\title{
SENSIBILIDAD DEL EFECTO DE ESTELA A LA ESTABILIDAD DE LA CAPA LÍMITE ATMOSFÉRICA EN UN PARQUE EÓLICO ONSHORE DE LA PATAGONIA ARGENTINA
}

Gonzalo Pablo Navarro Diaz ${ }^{1,2}$, María Laura Mayol $^{1,2}$, Andrea Celeste Saulo ${ }^{1,3}$ y Alejandro Daniel Otero ${ }^{4,2}$

${ }^{1}$ Departamento de Ciencias de la Atmósfera y los Océanos, FCEyN, UBA.

${ }^{2}$ Centro de Simulación Computacional para Aplicaciones Tecnológicas, CSC-CONICET.

${ }^{3}$ Servicio Meteorológico Nacional, Argentina.

${ }^{4}$ Facultad de Ingeniería, UBA.

(Manuscrito recibido el 30 de marzo de 2020, en su versión final el 22 de septiembre de 2020)

\section{RESUMEN}

En este trabajo se presenta un estudio de sensibilidad de la estela que se produce detrás de los aerogeneradores ante los distintos regímenes de estabilidad presentes en la capa límite atmosférica. Este análisis se lleva a cabo sobre un extenso registro de mediciones en uno de los parques eólicos más grandes de Argentina emplazado sobre tierra en la región patagónica. Se analizan datos tanto del período previo a su construcción como durante su etapa productiva. El enfoque resulta novedoso al analizar la dependencia del impacto negativo de la estela sobre la producción en función de los distintos regímenes de estabilidad que se desarrollan a lo largo del día y estacionalmente. Los resultados coinciden con las tendencias reportadas por otros autores, encontrando una disminución notable de la estela, y una mejora de la producción asociada, durante condiciones inestables. El menor impacto de las estelas se registra durante el verano en las horas del medio día, donde se dan típicamente estas condiciones. Contrariamente para condiciones estables, que ocurren generalmente durante la noche, el impacto de las estelas es mayor. El comportamiento promedio en general se aproxima a la condición neutral.

Palabras clave: energía eólica, efecto estela, capa límite atmosférica, regímenes de estabilidad.

\section{SENSITIVITY OF THE WAKE EFFECT TO THE ATMOSPHERIC BOUNDARY LAYER STABILITY IN AN ONSHORE WIND FARM IN THE ARGENTINEAN PATAGONIA}

\begin{abstract}
In this work, a sensitivity study of the downstream wakes produced by wind turbines for different stability regimes in the atmospheric boundary layer is presented. The analysis is made on an extensive measurement data set of one of the largest onshore wind farms located in the Patagonian region of Argentina. Data from periods before the construction and during production of the park are analyzed. The novelty of the approach is the analysis of the negative wake impact on production and its
\end{abstract}


dependence on the different stability regimes in the day and seasonal cycles. The outcomes coincide with the trends reported by other authors, finding a notable decrease in the wake impact during unstable conditions. The smallest impact is found during the summer at midday hours, when typically unstable conditions appear. On the contrary, during stable conditions, which generally occur at night, the impact is higher. The typical behavior of the park is in general close to the neutral condition.

Keywords: wind energy, wake effect, atmospheric boundary layer, stability.

\section{INTRODUCCIÓN}

El comportamiento de la parte de la atmósfera en contacto con la superficie terrestre, denominada capa límite atmosférica (CLA), es muy importante para la energía eólica. Esto es debido a que la zona de barrido de las aspas de los aerogeneradores abarca desde los primeros metros sobre la superficie terrestre hasta alturas de punta de pala de entre 200 y $300 \mathrm{~m}$. Este comportamiento tiene efecto sobre uno de los fenómenos más relevantes en el diseño y cálculo de la producción de los parques eólicos, el efecto de estela, el cual se produce aguas abajo de los aerogeneradores y tiene un impacto negativo en la producción (Navarro Diaz et al., 2019). Cuando el aire fluye a través de una turbina, ésta extrae energía del viento, produciendo una estela detrás de sí. Estas estelas se caracterizan por una reducción de la intensidad del viento y un aumento de la turbulencia. Si las estelas impactan total o parcialmente en otros aerogeneradores ubicados aguas abajo, la producción de energía en los mismos se verá reducida. Además, las condiciones de velocidad no homogéneas y la mayor turbulencia dentro de las estelas, disminuye la vida útil de aquellos aerogeneradores afectados.

La intensidad del efecto de estela depende tanto de las características estructurales y operativas de los aerogeneradores como así también de las condiciones turbulentas presentes en la CLA. Estos factores hacen que tanto $\mathrm{su}$ intensidad como su longitud aguas abajo varíe considerablemente. Si bien los aprovechamientos de la energía eólica generalmente corresponden a ubicaciones con recursos de vientos fuertes, donde la turbulencia tiene un origen predominantemente mecánico, eso no significa necesariamente que se puedan ignorar los efectos de origen térmicos, y varios autores han estudiado la influencia de la estabilidad atmosférica en las características de estela. Por ejemplo, Schepers et al. (2012) llevaron a cabo un análisis de sensibilidad de las estelas de un parque eólico sobre mar (offshore) para diferentes regímenes de estabilidad de la CLA, encontrando que para las condiciones estables, típicas durante la noche, la estela se mantiene intensa a lo largo de grandes distancias aguas abajo. Contrariamente, en condiciones inestables, generalmente alrededor del medio día, la mayor intensidad en la mezcla de la CLA acelera el desvanecimiento de la estela, reduciendo su impacto.

En la literatura se encuentran diferentes estudios del impacto de la estabilidad de la CLA sobre parques offshore. En cambio, los estudios sobre el impacto de dicho fenómeno en parques sobre tierra, (onshore), son escasos. Recientemente, Doubrawa et al. (2019) analizaron mediciones LIDAR del viento detrás de un solo aerogenerador para tres condiciones de estabilidad: cercana a la neutralidad, ligeramente inestable y muy estable. Por otro lado, en el trabajo de Han et al. (2018) se midió el perfil de la estela de un aerogenerador mediante dos mástiles meteorológicos provistos de anemómetros de coperolas a distintas alturas. Además, utilizaron un anemómetro sónico para estimar la estabilidad, pudiendo separar las mediciones de estela para los regímenes estable, inestable y neutral. $\mathrm{Al}$ 
igual que sucede en parques offshore, los autores de ambos trabajos encuentran que para condiciones inestables la estela se disuelve a distancias más cortas que para condiciones neutrales. Contrariamente, para condiciones muy estables la estela se mantiene intensa en una distancia mucho mayor aguas abajo y su ancho se mantiene constante. Ante este panorama, el estudio de la estabilidad sobre los parques onshore cobra una gran importancia, especialmente en Argentina, donde todos los parques eólicos actuales y previstos en el corto y mediano plazo serán construidos sobre tierra.

Es por ello que en este trabajo se propone analizar la interacción de estelas entre varios aerogeneradores en un parque eólico onshore bajo la influencia de las diferentes estabilidades atmosféricas asociadas al ciclo diurno y su variación estacional. Este análisis se lleva a cabo empleando las mediciones típicamente disponibles en un parque operativo: las variables registradas en el mástil meteorológico y la potencia de los aerogeneradores, consecuentemente, el cálculo de estabilidad será aproximado según los parámetros existentes. Se aborda el caso particular del Parque Eólico Rawson, uno de los parques de gran tamaño más antiguos de Argentina del cual se cuenta con una serie extensa de mediciones.

Este trabajo se organiza de la siguiente manera. En la sección 2 se describe la metodología para determinar de manera local los regímenes de estabilidad y los conjuntos de datos de mediciones del parque eólico que se han utilizado. Posteriormente, en la sección 3, se analiza cómo la variabilidad de los regímenes de estabilidad en la cercanía de los aerogeneradores influencia el desarrollo de la estela. Para ello, en una primera parte, se analizan las mediciones de viento antes de la construcción del parque, de las cuales se obtiene la distribución de los regímenes de estabilidad. Finalmente se trabaja con las mediciones de mástil meteorológico y potencia de los aerogeneradores en la etapa productiva del parque, con el fin de analizar el efecto de la estabilidad sobre las estelas a lo largo del ciclo diurno y para distintas estaciones del año.

\section{MÉTODOS Y DATOS}

\subsection{Metodología}

A lo largo del día se pueden registrar distintos regímenes de estabilidad de la CLA, los cuales se pueden dividir básicamente en tres: inestable, estable y neutral. Existen diferentes criterios para establecer el régimen en cada período de medición, los cuales incorporan una mayor o menor cantidad de variables y procesos atmosféricos (Sedefian y Bennett, 1980). El método más completo es el creado a partir de la Teoría de Semejanza de Monin-Obukhov (MOST) (Newman y Klein, 2014). De esta teoría se desprende el parámetro fundamental de estabilidad $\zeta=\mathrm{z} / \mathrm{L}$, donde $\mathrm{z}[\mathrm{m}]$ es la altura sobre el suelo y L [m] la longitud de Monin-Obukhov. En función de este parámetro $\zeta$ se definen los rangos de las distintas condiciones de estabilidad atmosférica. Lamentablemente, en la mayoría de los parques eólicos no se cuenta con mástiles meteorológicos con el instrumental preciso para la obtención de las variables necesarias para calcular dicho parámetro, a saber, los flujos turbulentos de cantidad de movimiento y calor. Un caso particular es el estudio de Cañadillas et al. (2011) realizado en el parque eólico of $f$ shore Alpha Ventus en Alemania, en donde se emplearon las mediciones de una plataforma marina experimental usando un anemómetro sónico.

Generalmente, los parques eólicos solo disponen de mástiles con anemómetros de coperolas, veletas y termómetros a varias alturas, y un barómetro. Estas alturas abarcan desde las cercanas al suelo hasta las equivalentes a la altura de góndola de los aerogeneradores, que es la altura del eje de rotación del rotor del aerogenerador (alrededor de 80 a $100 \mathrm{~m}$ ). Una alternativa para estimar la estabilidad atmosférica es a través del número de Richardson bulk o por diferencias finitas $\left(R_{i}\right)$, que se obtiene a partir del gradiente 
local de las mediciones promediadas en el mástil en períodos de 10 minutos. El $\mathrm{R}_{i}$ es un parámetro importante para la evaluación del efecto relativo de la estratificación térmica y la variación del viento con la altura. Dentro de sus aplicaciones, es fundamental para el transporte y dispersión de contaminantes a escalas locales y regionales (Stull, 2000). También se ha empleado para el estudio de la influencia de las condiciones de estabilidad sobre las estelas de los aerogeneradores en parques offshore (Dörenkämper et al., 2015). Este parámetro es un número adimensional y permite determinar la estabilidad a partir de la relación entre la producción o consumo de turbulencia debida al empuje térmico y la producción debida a los procesos friccionales con la superficie. Si se dispone de la temperatura y la velocidad del viento a dos alturas distintas, por ejemplo una cercana al suelo $\left(z_{1}\right)$ y otra al nivel de la góndola $\left(z_{2}\right)$, el valor de $R_{i}$ se calcula según (Bodine et al., 2009):

$$
R_{i}=\frac{\mathrm{g} \Delta z_{U}^{2}\left[\frac{T_{2}-T_{1}}{\Delta z_{T}}+\Gamma_{d}\right]}{T_{1}\left[U_{2}-U_{1}\right]^{2}}
$$

Donde $T_{1}, T_{2}[\mathrm{~K}]$ son las temperaturas medidas en los niveles inferior y superior de medición de esta variable. De manera análoga, $U_{1}$ y $U_{2}$ $[\mathrm{m} / \mathrm{s}]$ son las velocidades medidas en los niveles inferior y superior de medición de esta variable y $\Delta z_{U}$ y $\Delta z_{T}$ son las diferencias de altura [m] entre los niveles empleados para medir la velocidad y la temperatura, repectivamente. Los niveles de medición de ambas variables deben corresponderse lo mejor posible. $\Gamma_{d} \approx 0,01[\mathrm{~K} / \mathrm{m}]$ es la pendiente del perfil adiabático seco y $g$ la aceleración de la gravedad.

El número de $R_{i}$ puede tomar valores negativos (CLA inestable), positivos (CLA estable) o cercanos a cero (CLA neutral). En este trabajo se emplea la clasificación de los regímenes de estabilidad en función de $R_{i}$ sugerida en el estudio de Newman y Klein (2014), según se especifica en la tabla I. Si bien en dicho trabajo utilizan niveles diferentes, los límites del rango para atmósferas neutrales de Newman y Klein (2014) se basan en el limite adoptado por
Mauritsen y Svensson (2007) quienes trabajan con diferentes combinaciones de niveles, algunas bastante más cercanas a las del presente trabajo. Por otro lado, Dörenkämper et al. (2015) en una aplicación afín utilizando niveles en alturas ligeramente mayores a las aquí utilizadas, definen el rango para la clasificación neutral de la atmósfera como $-0,15<R_{i}<0,15$, es decir más amplio que el adoptado por Newman y Klein (2014), lo que conduciría a clasificar más situaciones dentro del rango neutral. Por último, cabe destacar que Newman y Klein (2014) quienes analizan la estabilidad en una ubicación sobre tierra al igual que en este trabajo, obtienen resultados consistentes a los presentados más adelante.

\begin{tabular}{|l|c|}
\hline Clasificación de estabilidades & $R i$ \\
\hline Muy inestable & $R i<-0,2$ \\
\hline Inestable & $-0,2 \leq R i<-0,1$ \\
\hline Neutral & $-0,1 \leq R i<0,1$ \\
\hline Estable & $0,1 \leq R i<0,25$ \\
\hline Muy estable & $0,25 \leq R i$ \\
\hline
\end{tabular}

Tabla I: Límites de la clasificación de estabilidades en la CLA en función de Ri (Newman y Klein, 2014).

Como indicador del impacto de las estelas, se utiliza la relación de potencias medias entre los aerogeneradores afectados y no afectados por estelas, de manera análoga al análisis de Schepers et al. (2012):

$$
\mathrm{R}_{P}=\frac{P_{T_{1}}}{P_{T_{2}}}
$$

donde $T_{1}$ es el aerogenerador afectado por las estelas aguas abajo y $T_{2}$ aquel no afectado, aguas arriba. Este indicador de impacto se evalúa para distintas direcciones de viento entrante medidas en el mástil.

\subsection{Fuentes de mediciones}

En este estudio se hará foco en el Parque Eólico Rawson (PER). El mismo está emplazado en la Patagonia Argentina a una altitud de $150 \mathrm{~m}$ y a $10 \mathrm{~km}$ de la costa. Este parque fue construido en tres etapas; la primera fue inaugurada en 
octubre del 2011 y contó con 27 aerogeneradores Vesta V90, con una potencia nominal de 1,8 MW. La segunda etapa fue terminada en enero del 2012, en la cual se sumaron 16 aerogeneradores de las mismas características, llegando a un total de 77,4 MW. Por último, la tercer etapa fue inaugurada en diciembre del 2017, agregando 12 aerogeneradores Vestas V100 de 2,0 MW cada uno. En total suma 55 aerogeneradores y una capacidad instalada de 101,4 MW, siendo uno de los parque eólicos más grande de Argentina.

Previo a la construcción del parque eólico, se llevó a cabo una valoración del recurso eólico en el terreno disponible, instalando una torre meteorológica en el centro del parque actual. Este mástil central funcionó desde octubre del 2010 hasta mediados de septiembre del 2011, un total de casi 1 año de mediciones continuas. El mismo estaba compuesto por tres anemómetros de coperolas NRG \#40 (a 40, 60 y $80 \mathrm{~m}$ ), dos veletas NRG \#200P (78 y $58 \mathrm{~m})$, dos sensores de temperatura NRG \#110S (a 3 y $79 \mathrm{~m})$ y un barómetro NRGBP-20 a $1 \mathrm{~m}$. En cada instrumento se registraron mediciones promediadas en períodos de 10 min y, además, se cuenta con los valores del desvío estándar de la intensidad y de la dirección de viento en los mismos períodos. Con estas variables es posible el cálculo del número de Richardson bulk $\left(R_{i}\right)$ como indicador de la estabilidad de la CLA cercana a los aerogeneradores. Debido a que se dispone de la temperatura cercana al suelo y a la altura de góndola, pero la velocidad de viento solo a la altura de góndola, se decide emplear la formula de $R_{i}$ asumiendo que $U_{1}=0 \mathrm{al}$ no disponerse del dato en el nivel más bajo. Esta suposición podría dar como resultado una sobrestimación de la cortante vertical del viento y consecuentemente una subestimación del $R_{i}$.

Además de la información del estudio previo, en este trabajo se utilizan datos operativos del PER en sus dos primeras etapas. Hasta ese momento, el parque estaba compuesto de 43 aerogeneradores Vestas V90, con una altura de góndola de $80 \mathrm{~m}$ y un diámetro del rotor $\mathrm{D}$
$=90 \mathrm{~m}$, distribuidos en 4 filas en dirección aproximadamente $\mathrm{SO}$, separados a $4 \mathrm{D}$ dentro de la fila y a $12 \mathrm{D}$ entre filas. Esto da al parque una dimensión total de $4 \mathrm{~km} \mathrm{x} 4$ $\mathrm{km}$. Además, el parque cuenta con un mástil meteorológico localizado en la esquina NO y a $6 \mathrm{D}$ del rotor más cercano. El mismo se instaló posteriormente al estudio de prefactibilidad del parque, en febrero del 2011, desactivando el mástil central del estudio previo. La disposición de los aerogeneradores y el mástil meteorológico en el PER se muestra en la figura 1. A diferencia de la anterior torre central, este mástil cuenta con dos anemómetros VAISALA WAA151 (a 35 y $80 \mathrm{~m}$ ), una veleta VAISALA WAA151 a $79 \mathrm{~m}$ y un sensor de temperatura y presión a $78 \mathrm{~m}$. De esos dos anemómetros, se decide trabajar con el que está ubicado a $80 \mathrm{~m}$, dado que corresponde a la altura de la góndola de los aerogeneradores. $\mathrm{Al}$ igual que en el mástil del estudio previo, en éste se registran las variables promediadas en períodos diez-minutales. También se utiliza la intensidad turbulenta longitudinal $\left(I T_{u} \equiv \frac{\sigma_{u}}{U}\right)$ calculada en base al desvío estándar reportado por el anemómetro cada $10 \mathrm{~min}$, lo cual es equivalente a considerar solo el desvío estándar de las componentes horizontales de la velocidad. La comparación entre esta intensidad turbulenta y la calculada en base a las tres componentes de la velocidad y su validez para determinar la estabilidad de la CLA fue realiza por Wharton y Lundquist (2012), demostrando que la $I T_{u}$ se puede emplear como un indicador de la estabilidad atmosférica y de la mezcla de la estela.

Para el análisis de datos del mástil meteorológico durante el período de producción se eligió trabajar a partir de la etapa 2, abarcando desde comienzos de enero de 2012 hasta fin de abril de 2018 (6 años y 4 meses). De esta base de datos se descartó el período desde febrero del 2014 hasta julio 2015 inclusive (1 año y 6 meses) debido a que la empresa informa que los instrumentos del mástil estuvieron descalibrados. Con esta supresión de datos, se cuenta con un total de 4 años y 10 meses de mediciones. A estas mediciones se les aplicó un 


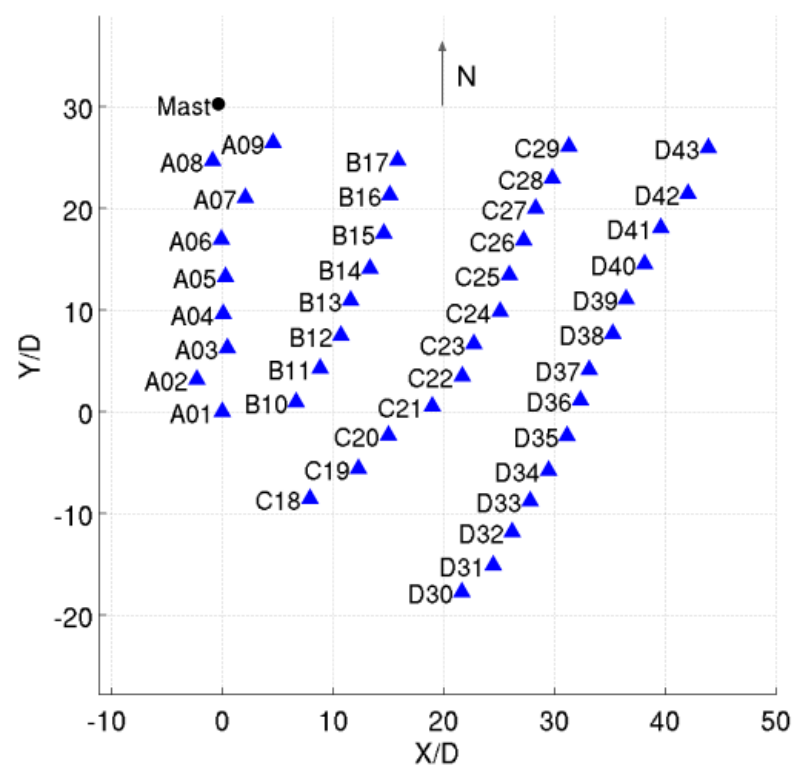

Figura 1: Disposición de los aerogeneradores, con su identificación por fila A, B, C y D, y del mástil meteorológico. Las dimensiones horizontales están adimensionalizadas con el diámetro del aerogenerador $(\mathrm{D}=90 \mathrm{~m})$.

filtro para descartar los períodos de 10 minutos que tuvieran faltante de datos en alguno de los instrumentos de medición. Esto resulta en que finalmente se cuente con el $93,4 \%$ de los datos, aproximadamente 238 mil mediciones de 10 minutos cada una o un equivalente a 4 años y 7 meses. Cabe destacar que en el segundo capítulo de Waimann (2016), se efectuó un análisis de las características del viento en PER, pero su muestra fue acotada a un período total, antes de filtrar por faltantes de datos, de 2 años.

Para el análisis de la producción de los aerogeneradores se parte del mismo conjunto de datos filtrados de la torre meteorológica. Los aerogeneradores reportan mediante su sistema de control la potencia media generada en períodos diez-minutales. Se debieron descartar los datos a partir de julio del 2017, debido a que las curvas de potencia de todos los aerogeneradores fueron modificadas por el fabricante, aumentando su potencia nominal. De esta manera, los datos utilizados corresponden al período anterior a julio del 2017 y, por lo tanto, todos los aerogeneradores tienen en todo momento la misma potencia nominal $(1.8 \mathrm{MW})$. Finalmente, se descartaron los períodos donde faltasen mediciones de alguno de los aerogeneradores, contándose con aproximadamente 32 mil datos diez-minutales. En definitiva, el volumen total de datos, luego de filtrar las mediciones del mástil y de los aerogeneradores equivale a 1 año 7 meses.

Para analizar el impacto de estelas, se eligió trabajar con las mediciones de mástil meteorológico y producción de los cuatro aerogeneradores más cercanos al mismo (A6, A7, A8 y A9), figura 2, con el fin de lograr una buena correlación entre las mediciones del mástil y la producción. Los aerogeneradores A8 y A7 están separados a $4,7 \mathrm{D}(423 \mathrm{~m})$ y se alinean para la dirección de viento $320^{\circ}$, quedando $\mathrm{A} 7$ afectado por la estela de A8. Otro caso de interferencia se puede identificar cuando A7 y A6 quedan dentro de la estela del A9. Los A7 y A6 están ubicados a $5,7 \mathrm{D}(513 \mathrm{~m})$ y $10,5 \mathrm{D}(945 \mathrm{~m})$ aguas abajo del A9 a lo largo de la dirección $25^{\circ}$. 


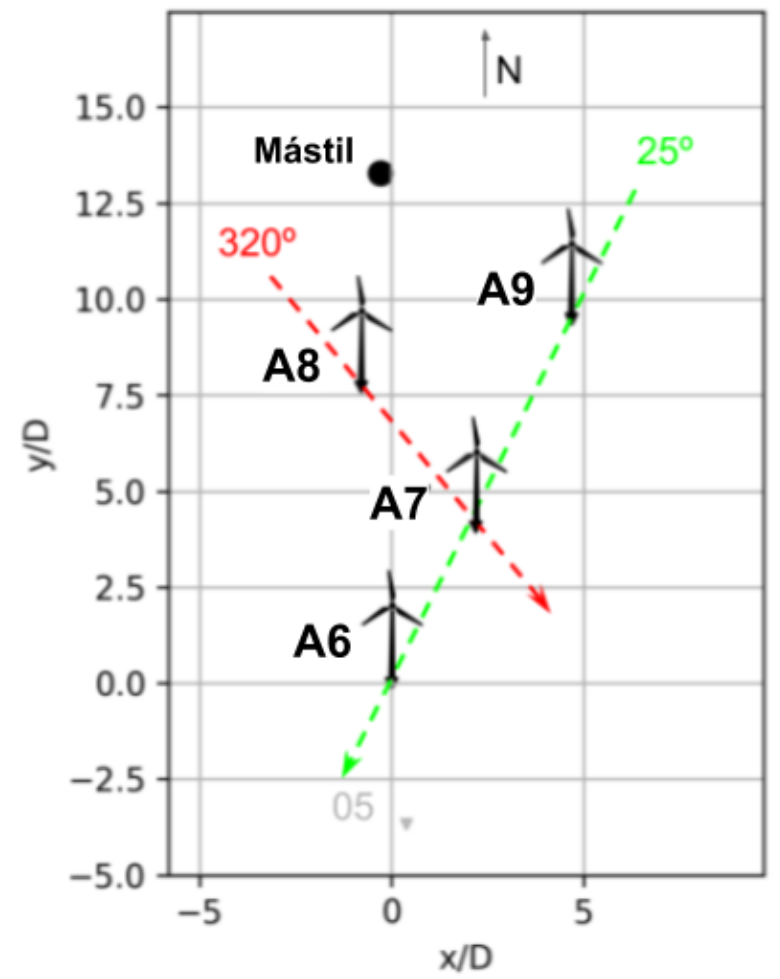

Figura 2: Ubicación de los 4 aerogeneradores más cercanos al mástil meteorológico del PER.

\section{RESULTADOS, ANÁLISIS Y DISCUSIÓN}

\subsection{Características de la distribución de velocidad y dirección}

Una vez realizado el filtrado de los datos, se lleva a cabo el análisis de las mediciones de viento del mástil meteorológico del estudio previo y durante la etapa productiva del parque eólico, con el fin de obtener una caracterización de régimen de viento local. En la figura 3 se presentan las rosas de viento, generadas a partir de las mediciones en ambas etapas. Allí puede verse que el comportamiento general resulta equivalente. Como datos principales, se obtiene que los vientos predominantes tienen componente oeste, con una mayor frecuencia en la dirección $282^{\circ}$ para el estudio previo y $265^{\circ}$ durante el período productivo. Esta dirección predominante también fue registrada en el trabajo de Cúneo et al. (2018), en el cual se analizaron las mediciones a $10 \mathrm{~m}$ de una torre en la estación meteorológica que el Servicio Meteorológico Nacional posee en Trelew, ubicada a $18 \mathrm{~km}$ del PER. En la etapa productiva, cuando el viento sopla desde la dirección oeste, el mástil del parque se encuentra libre de la interferencia de la estela de los aerogeneradores, por lo que la influencia de este efecto es mínimo en el análisis de datos. Desafortunadamente para un cierto rango de direcciones $\left(100^{\circ}\right.$ a $\left.200^{\circ}\right)$ el mástil se encuentra parcial o totalmente interferido por la estela del parque, lo que corresponde en este caso a un $17,2 \%$ de los datos.

Se ha encontrado que la distribución de Weibull proporciona una buena representación de la variación en la velocidad del viento media por hora durante un año en muchos sitios típicos (Seguro y Lambert, 2000) y también vale para el PER en el período estudiado por Waimann (2016). Esta distribución toma la forma

$$
F(U)=\exp \left(-\left(\frac{U}{c}\right)^{k}\right)
$$

donde $F(U)$ es la fracción de tiempo para la cual la velocidad media del viento por hora excede el valor de velocidad de viento $U$. Está caracterizada por dos parámetros, un parámetro de escalacy un parámetro de formakque describe la variación alrededor de la media.cestá relacionado con la velocidad media anual del viento $\bar{U}$ a través de la relación

$$
\overline{\mathrm{U}}=c \Gamma\left(1+\frac{1}{k}\right)
$$

donde $\Gamma$ es la función gamma. En la figura 4 se muestran los histogramas de la velocidad de viento media por hora con los respectivos ajustes a la distribución Weibull para ambas etapas de estudio. Se verifica que el comportamiento general en ambas etapas resulta equivalente. Las respectivas velocidades promedio son de $8,19 \mathrm{~m} / \mathrm{s}$ y 7,58 $\mathrm{m} / \mathrm{s}$ a $80 \mathrm{~m}$. Se registran con frecuencia casi nula valores de velocidad superiores a la 

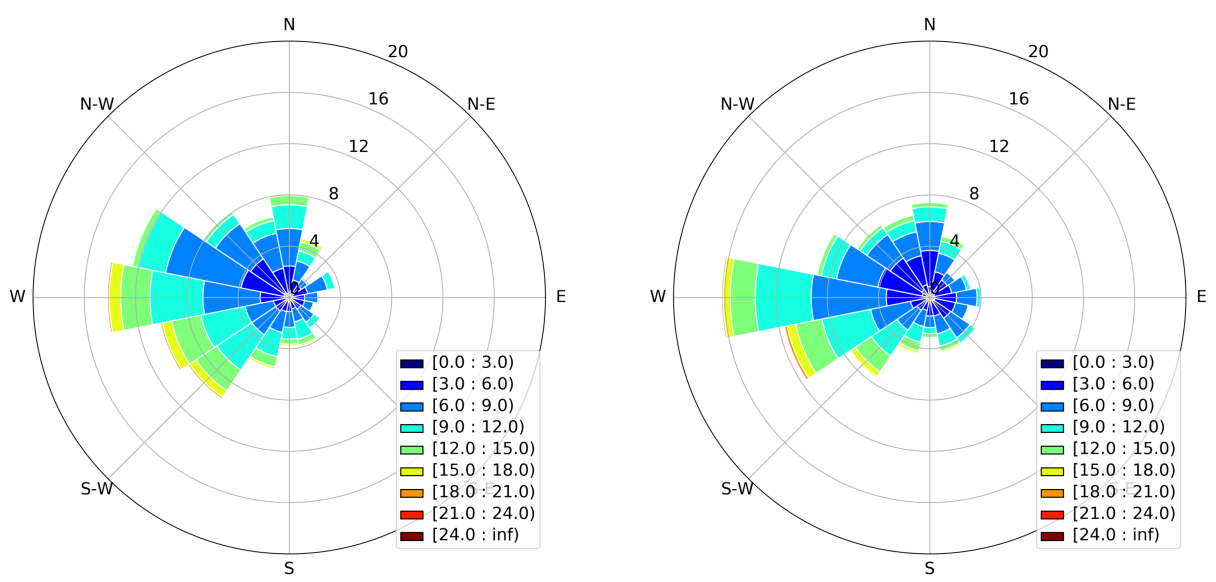

Figura 3: Rosas de vientos discretizadas en 16 sectores, correspondientes a la ubicación del PER durante dos períodos de medición: (izquierda) durante el estudio previo y (derecha) en la etapa productiva. Se utilizaron las mediciones de los mastiles con instrumentos a $80 \mathrm{~m}$ de altura. Velocidades en $[\mathrm{m} / \mathrm{s}]$.

velocidad de cut-out en la cual el aerogenerador se detiene por exceso de velocidad, y que para el caso del PER corresponde a 25 $\mathrm{m} / \mathrm{s}$. Estos resultados son consistentes con los de Waimann (2016), indicando que la frecuencia observada de los rangos entre 5 y $8 \mathrm{~m} / \mathrm{s}$ exceden a los que representa la distribución teórica, aunque en términos generales, esta representa razonablemente bien el comportamiento observado.

\subsection{Regímenes de estabilidad en Rawson}

Con el objetivo de caracterizar los regímenes de estabilidad en la ubicación del PER, se realiza el análisis de las mediciones del mástil meteorológico emplazado para confeccionar el estudio previo del recurso eólico. Este mástil cuenta con sensores de temperatura a dos alturas, lo cual resulta fundamental para la estimación de la estabilidad en la CLA utilizando el $R_{i}$. Las características generales del recurso observado en esta etapa se presentan en los paneles izquierdos de las figuras 3 y 4 , donde se muestran respectivamente la rosa de vientos y la distribución de velocidades.

En la figura 5 (arriba) se muestra la distribución de casos en función del valor $\operatorname{del} R_{i}$. Se puede observar que la mayoría de los datos se agrupan cerca de $R_{i}=0$, correspondiente al régimen neutral. Para valores menores o mayores a $R_{i}=0$, la distribución decae monótonamente. Con respecto a la distribución de categorías de estabilidad en función de la velocidad del viento a la altura de la góndola, en la figura 5 (abajo) podemos ver que para velocidades bajas, menores a $5 \mathrm{~m} / \mathrm{s}$, existe un predominio de las CLA extremadamente estables o inestables. Para velocidades más altas, las estabilidades cercanas a la neutralidad comienzan a tener una mayor preponderancia, mientras que para velocidades por encima de la velocidad nominal de los aerogeneradores instalados en PER 1 y 2 $(15 \mathrm{~m} / \mathrm{s})$ solo se registran regímenes neutrales. Entre las velocidades de 5 y $9 \mathrm{~m} / \mathrm{s}$ existe una cantidad considerable de cada una de las 5 clases de estabilidades. Por esto, en la sección 3.3 se utilizará este rango de velocidades para el análisis de las estelas de los aerogeneradores bajo el efecto de las diferentes estabilidades.

Para tener una visión general, en la figura 6 se muestra la proporción del tiempo en que ocurre cada uno de los 5 regímenes de estabilidad. Se puede observar que aproximadamente la 

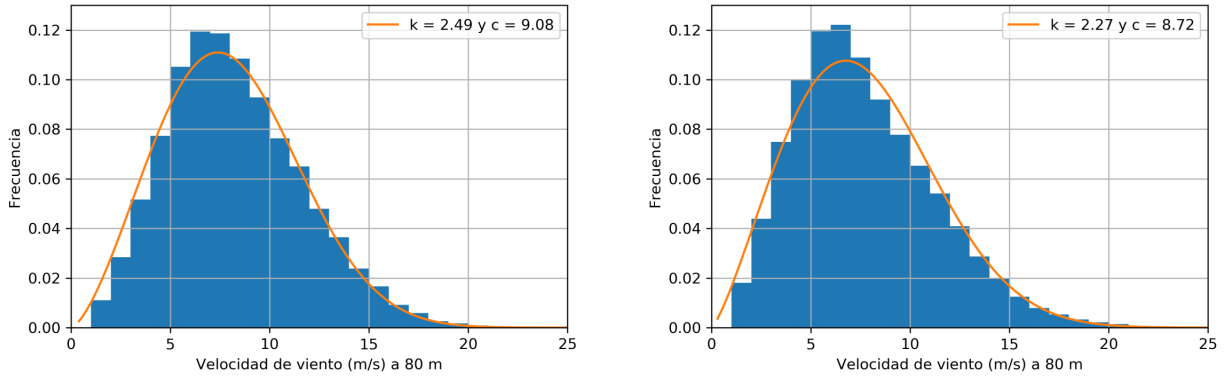

Figura 4: Distribución de datos diez-minutales de velocidad de viento, correspondientes a la ubicación del PER durante dos períodos de medición: (izquierda) durante el estudio previo y (derecha) en la etapa productiva. Se utilizaron las ediciones de los mástiles con instrumentos a $80 \mathrm{~m}$ de altura. También se grafican las correspondientes distribuciones Weibull ajustadas a los datos y se reportan sus coeficientes. Velocidades en $[\mathrm{m} / \mathrm{s}]$.

mitad del tiempo $(56,1 \%)$ se registra una CLA neutral, seguido por una mayor cantidad de casos estables $(13,1 \%+17 \%=30,1 \%)$ que de inestables $(7,8 \%+6 \%=13,8 \%)$.

En la figura 7 se presenta el ciclo diurno del promedio anual de $R_{i}$ para cada hora. Los datos fueron seleccionados para el rango de velocidades de funcionamiento del aerogeneradores (de 4 a $25 \mathrm{~m} / \mathrm{s}$ ) con el fin de encontrar una relación con el comportamiento de la estela. Se puede observar que el valor de $R_{i}$ tiende a tomar valores positivos (estables) durante el período nocturno (de 19 a 8 hs) y valores negativos (inestables) durante el período diurno (de 8 a $19 \mathrm{hs}$ ). Si tomamos el rango de horas durante el cual todo el año es de noche (23 a 4 hs) o de día (11 a 16 hs), obtenemos valores promedio de $R_{i}=0,17$ y $R_{i}=-0,09$, respectivamente.

Con el fin de profundizar el entendimiento de la dinámica de las estabilidades en el ciclo diurno, en la figura 8 se muestra la proporción de los 5 regímenes de estabilidad para cada hora, a partir de los mismos datos utilizados en la figura anterior. Se puede confirmar la tendencia a la estabilidad por la noche dado que en cerca del $45 \%$ de las veces la estabilidad queda dentro de la clasificación neutral, y el resto es exclusivamente estable o muy estable con mayor frecuencia de esta última. En comparación, para el día la cantidad de casos neutrales es mayor al $50 \%$, resultando el resto de los casos inestables o muy inestables. Esta tendencia a la estabilidad durante el período nocturno y la inestabilidad durante el diurno será clave para el análisis de comportamiento de la estela en ambos rangos horarios. Este análisis de los regímenes a lo largo del ciclo diurno nos permite asumir que durante los dos rangos horarios seleccionados, nocturno y diurno, la CLA tendrá un comportamiento mayoritariamente estable o inestable, respectivamente, tendiendo el período inestable a un promedio más cercano a la neutralidad. Por esta razón, se emplea este criterio para analizar el efecto sobre las estelas en la próxima sección, dado que el mástil utilizado en la etapa operativa del PER no cuenta con instrumental que permita calcular el $R_{i}$.

\subsection{Efecto de estabilidad en la interacción de aerogeneradores}

A continuación, con el fin de analizar el efecto de los regímenes de estabilidad en las estelas de los aerogeneradores, se realizará una comparación de la relación de potencias (ecuación 2) para distintos aerogeneradores cuando se encuentran afectados por las estelas de uno o varios aerogeneradores aguas arriba. Los períodos nocturnos y diurnos se dividieron, además, entre los meses de verano (DEF) e invierno 

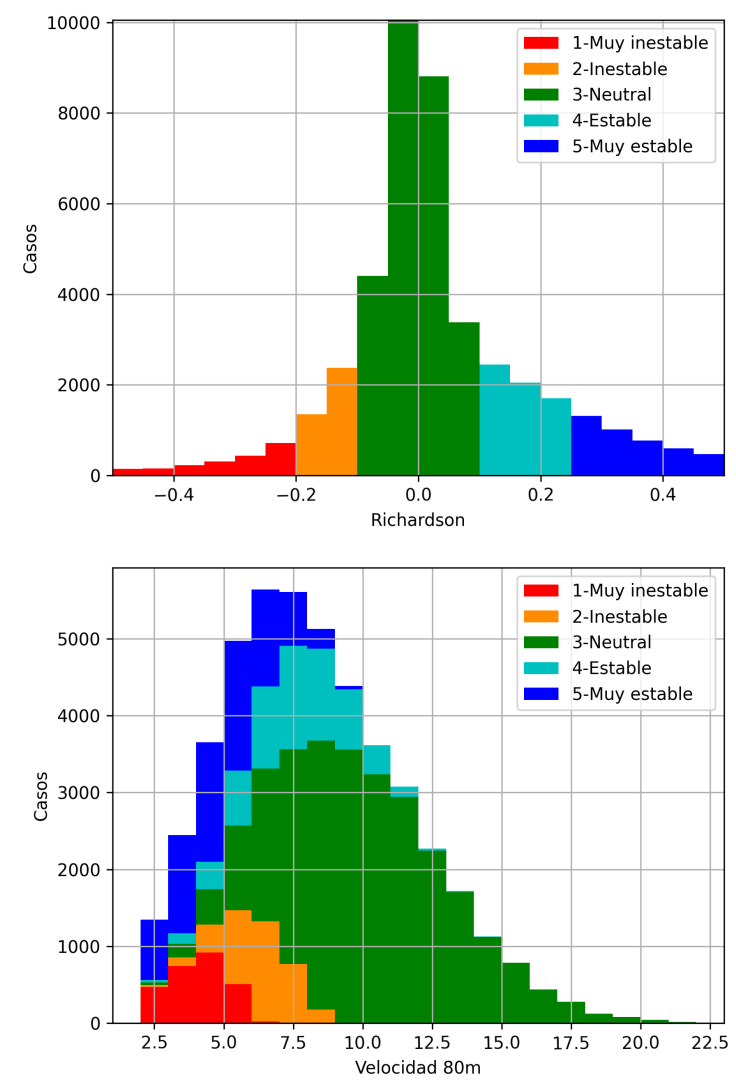

Figura 5: Distribución de casos en función de valor de $\mathrm{R}_{i}$ (arriba) y de la velocidad del viento a la altura de la góndola $80 \mathrm{~m}$ (abajo). Se indican los 5 regímenes de estabilidad mediante la clasificación de acuerdo al $\mathrm{R}_{i}$.

(JJA), con el fin de evaluar la influencia de los diferentes regímenes de estabilidad fuertemente estables e inestables sobre las estelas. Del conjunto de datos, se trabaja con las mediciones entre 5 y $9 \mathrm{~m} / \mathrm{s}$, que corresponden al rango en el cual la velocidad está por encima del valor de arranque y el aerogenerador aplica el mismo efecto de resistencia sobre el fluido dado que el coeficiente de empuje $C_{T}$ se mantiene aproximadamente constante. De esta manera, al considerar velocidades para las cuales los aerogeneradores tienen el mismo $C_{T}$, el déficit de potencia que se produce sobre el aerogenerador afectado por la estela de aquellos aguas arriba será independiente de la velocidad en el mástil, pero sí dependerá de la estabilidad

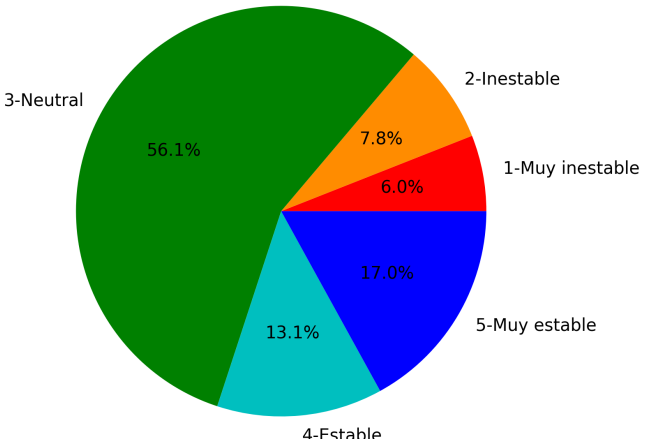

Figura 6: Proporción de ocurrencia de los 5 regímenes de estabilidad.

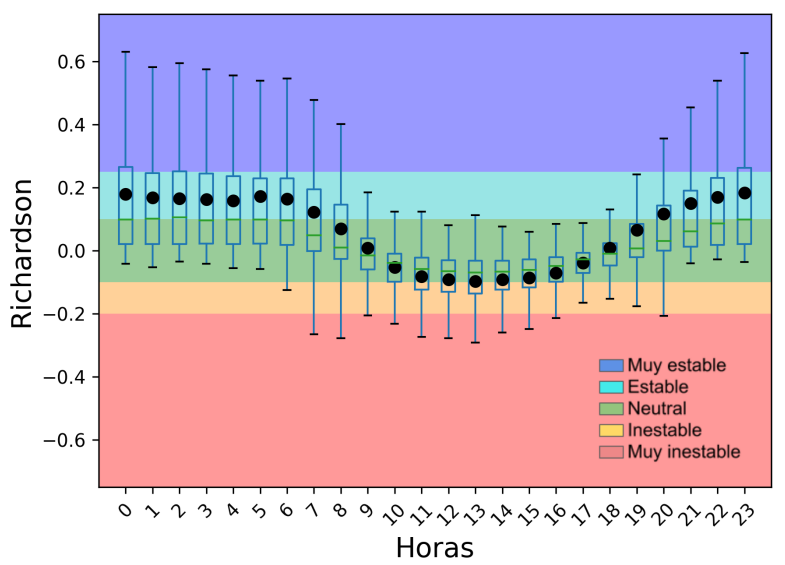

Figura 7: Variación del valor promedio anual de Ri para cada hora del ciclo diurno (marcadores negros). También se grafica el boxplot con el rango intercuartil (del primero al tercer cuartil). Se grafican los valores extremos del primer y cuarto cuartil, descartando como valores atípicos (outliers) aquellos que se alejan de los límites del boxplot más de 1,5 veces el rango intercuartil.

de la CLA. La dependencia respecto de este último factor será clave para el análisis de sensibilidad de las estelas a la estabilidad de la atmósfera.

$\mathrm{Al}$ reducir el rango de velocidades se modifican las proporciones de las diferentes estabilidades 


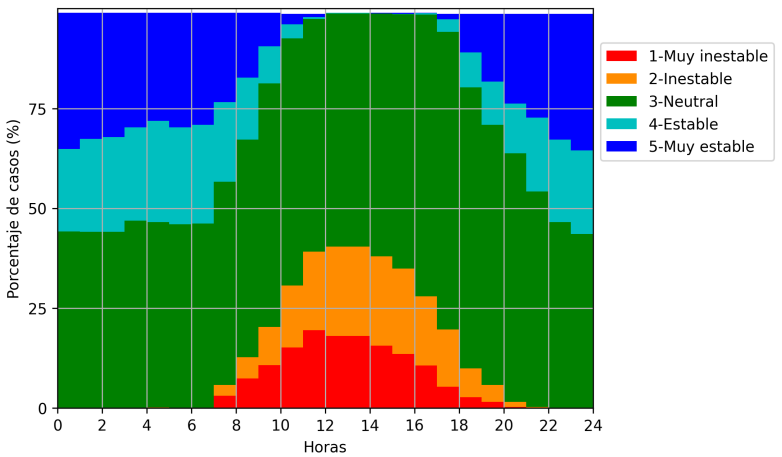

Figura 8: Porcentaje de los 5 regímenes de estabilidad para cada hora del ciclo diurno. Los datos fueron seleccionados para el rango de velocidades del funcionamiento del aerogenerador (4 a $25 \mathrm{~m} / \mathrm{s}$ ).

y el $R_{i}$ promedio respecto del rango completo de velocidades de trabajo del aerogenerador referido en la sección anterior. En la tabla II se presentan los porcentajes de ocurrencia de veces en que se detectan las distintas estabilidades en el rango de velocidades entre 5 y $9 \mathrm{~m} / \mathrm{s}$ durante los periodos diurno y nocturno. Claramente en el período diurno, la atmósfera es mayormente neutral con tendencia a la inestabilidad con un valor de $R_{i}$ promedio de $-0,11$. Por otro lado, en el período nocturno prácticamente la mitad de las ocurrencias corresponden a situaciones neutrales y el resto tiende a ser estable resultando en un valor de $R_{i}$ promedio de 0,21. Considerando el total de datos en el rango reducido de velocidades el comportamiento neutral resulta el más frecuente con tendencia a los regímenes estables, resultando un $R_{i}$ promedio de 0,08 .

\begin{tabular}{|l|r|r|r|}
\hline Clasificación de estabilidades & Diurno & Nocturno & Total \\
\hline Muy inestable & $8,2 \%$ & $0,0 \%$ & $2,4 \%$ \\
\hline Inestable & $43,6 \%$ & $0,0 \%$ & $14,7 \%$ \\
\hline Neutral & $47,9 \%$ & $29,4 \%$ & $44,6 \%$ \\
\hline Estable & $0,2 \%$ & $35,4 \%$ & $20,3 \%$ \\
\hline Muy estable & $0,1 \%$ & $35,2 \%$ & $18,0 \%$ \\
\hline
\end{tabular}

Tabla II: Proporciones de los distintos regímenes de estabilidad atmosférica para velocidades entre 5 y $9 \mathrm{~m} / \mathrm{s}$ durante los períodos diurno, nocturno y el total.
En la figura 9 (arriba) se presenta la relación de potencias entre los aerogeneradores A7 y A8, mientras que en las figuras 9 (medio) y 9 (abajo) se muestra la relación de potencias de A7 con respecto a $\mathrm{A} 9$ y $\mathrm{A} 6$ con respecto a A9. Las mediciones fueron promediadas en direcciones cada $4^{\circ}$ para un rango de $+/-25^{\circ}$ respecto de la dirección en que se alinean las turbinas. En los tres casos se puede observar que se repite el patrón de diferencias entre el impacto del período diurno y nocturno, identificando a la zona de máxima interferencia como la más sensible a estos cambios de estabilidades. En esa zona en particular, la diferencia promedio entre la relación de potencias para ambos períodos es del $16 \%$ en la figura 9 (arriba), y del $24 \%$ para las figuras 9 (medio) y 9 (abajo).

Estos valores son similares a los hallados por (Schepers et al., 2012), quien encontró una diferencia del $20 \%$ entre el día y la noche para dos aerogeneradores distanciados a 3,8D. Esta clara disminución en el máximo de interferencia en presencia de inestabilidades se ve acompañado de un aumento de la dispersión angular de las curvas; esto sugiere que la mayor mezcla turbulenta estaría incrementando la dispersión horizontal de la estela, haciendo aumentar su ancho. Como una observación general, el período nocturno tiene un comportamiento similar al promedio general del día entero, con una relación de potencias ligeramente menor. Contrariamente, los resultados del período diurno se alejan mucho del promedio general, mostrando resultados de interferencias menores. Es importante notar que la diferencia entre los resultados se vuelve más marcada cuando los aerogeneradores se encuentran más distanciados unos de otros, como el caso de A7 y A9, o cuando se superpone el efecto de dos estelas como en el caso de A6 bajo el efecto de las estelas de A9 y A7. Estos cambios en la relación de potencias son debidos a que el impacto de estela depende de las condiciones de estabilidad en la CLA. Para atmósfera inestables, predominantemente cerca del medio día, la estela está sometida a una mayor mezcla, haciendo que su impacto sobre 

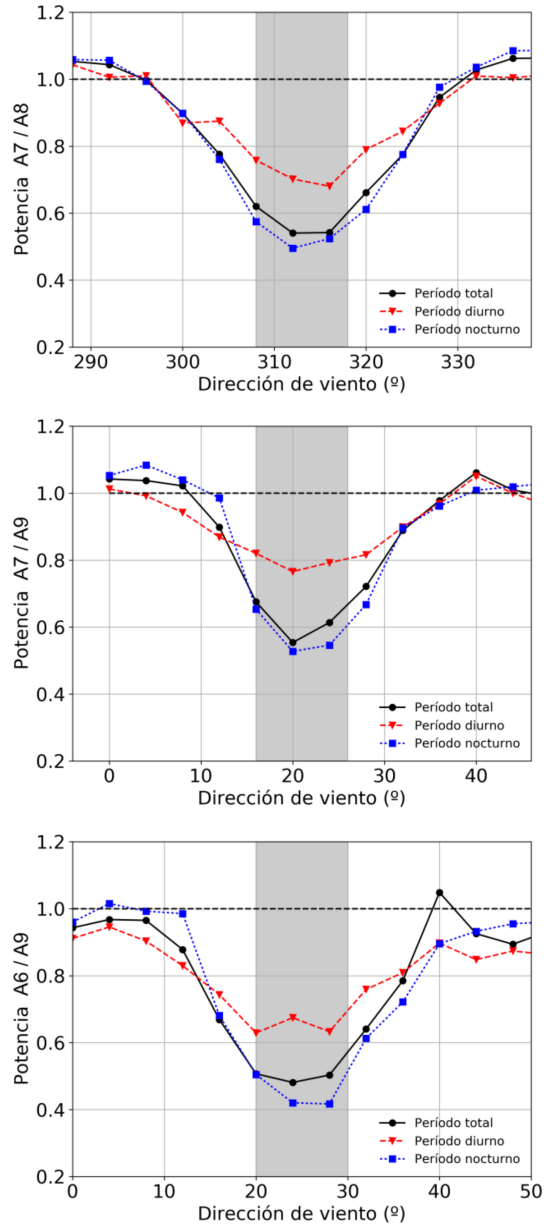

Figura 9: Impacto de las estelas en la potencia medida en términos de la dirección de viento para velocidades de entrada entre 5 y $9 \mathrm{~m} / \mathrm{s}$ para el período completo y su separación entre los rangos diurno y nocturno: (arriba) Aerogenerador A7 con respecto a A8 (dirección de referencia de $313^{\circ}$ ), (medio) aerogenerador A7 $\left(21^{\circ}\right)$ y (abajo) aerogenerador A6 $\left(25^{\circ}\right)$ con respecto al A9.

el aerogenerador aguas abajo sea más débil y su producción no se vea tan comprometida. A fin de ejemplificar el efecto de las distintas condiciones de estabilidad en la generación eólica, mencionaremos la situación particular en que el aerogenerador A7 se encuentra en la estela del aerogenerador A8 con viento de la dirección de $R_{P}$ mínima. En esta situación, para una velocidad de $8 \mathrm{~m} / \mathrm{s}$ (cercana al promedio del PER) el A8 entregaría una potencia de $880 \mathrm{~kW}$. Mientras tanto el A7 en condiciones promedio entregaría un $58 \%$ de esa potencia, es decir $510 \mathrm{~kW}$. En la situación promedio del rango diurno, el A7 produciría un $73 \%$ de la potencia de $\mathrm{A} 8$, es decir $642 \mathrm{~kW}$, superando en $132 \mathrm{~kW}$ el caso promedio. Por otro lado, en la situación promedio del rango nocturno, el A7 entregaría un $53 \%$ de la potencia de A8, es decir $466 \mathrm{~kW}$, una disminución de $44 \mathrm{~kW}$ respecto del caso promedio.

Debido a que el valor de mínima relación de potencias es el más sensible a los cambios de estabilidad, se estudiará su variación a lo largo del ciclo diurno a fin de analizar como éste afecta la interferencia de las estelas. Para evitar problemas debidos a la escasez de datos medidos en determinados momentos se utiliza como indicador de este valor mínimo de la relación de potencias el promedio de los valores en un rango de $+/-5^{\circ}$ alrededor de la dirección en que se alinean ambas turbinas. En la figura 10 se muestra la evolución de la relación de potencias mínima del aerogenerador A7 respecto del A8 a lo largo del ciclo diurno, separando el conjunto de datos entre los meses de invierno y verano. Se puede observar que durante el invierno la relación de potencias se mantiene con valor bajo a lo largo de todo el día. Contrariamente, durante el verano existe una gran diferencia entre las horas de noche y de día. En particular para las horas del medio día el impacto de la estela se vuelve muy débil, llegando la potencia del aerogenerador afectado a valores cercanos al $95 \%$ de la potencia del aerogenerador aguas arriba. Es necesario recordar que el indicador de la relación de potencias se calcula mediante el promedio del rango de $+/-5^{\circ}$ alrededor de la dirección de máxima interferencia; por lo tanto, este valor podría ser menor en la dirección exacta de alineamiento de ambas turbinas.

Una forma de explicar esta gran variabilidad en el impacto de la estela es a través de la intensidad turbulenta $I T_{u}$ medida en el anemómetro del mástil a $80 \quad \mathrm{~m}$. En 


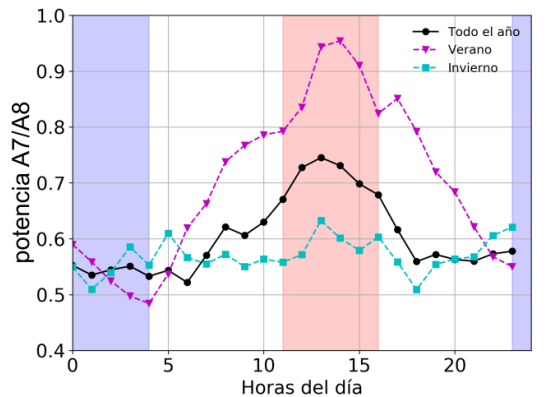

Figura 10: Relación de potencias mínima del aerogenerador A7 comparado con A8 a lo largo del día, separando el conjunto de datos entre los meses de invierno (JJA) y verano(DEF). Las zonas coloreadas en azul y rojo corresponden a las franjas horarias tomadas para el período nocturno y diurno, respectivamente.

la figura 11 se puede observar que este indicador de la turbulencia, a pesar de no considerar la turbulencia vertical, logra captar la variabilidad diurna y su distinción entre verano e invierno. Resulta notable como se repiten las características del comportamiento observado en las figuras 10 y 11 respecto del ciclo diurno y anual. De esta manera, se confirma que la variabilidad en el impacto de la estela es debida a la turbulencia, de origen mecánico y térmico, presente en la CLA.

\section{CONCLUSIONES}

En este trabajo se llevó a cabo un análisis del efecto de los distintos regímenes de estabilidad atmosférica en la producción de los parques eólicos afectando la interacción de las estelas de los aerogeneradores. Este estudio resulta novedoso al haberse realizado sobre las mediciones de mástil meteorológico y potencia de los aerogeneradores en un parque eólico onshore emplazado en la Patagonia, una de las regiones con mejor recurso eólico del mundo. Asimismo, se destaca la extensión del registro observacional, que es atípico para este tipo de análisis.

Al análisis del efecto de las estelas se le dio

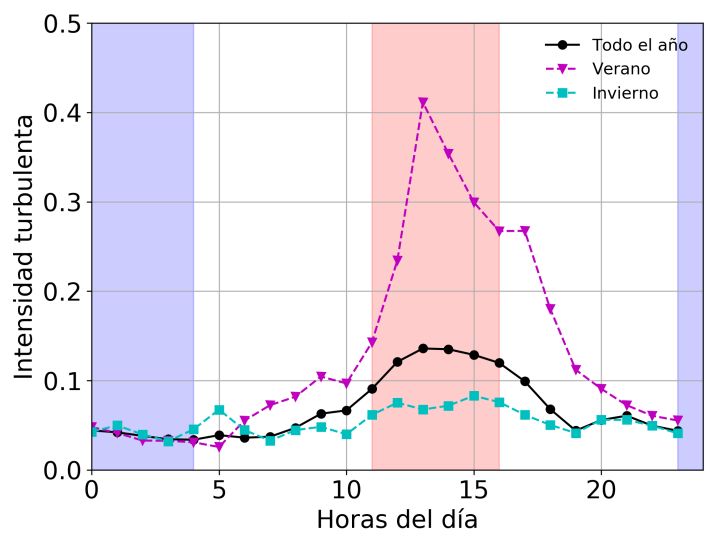

Figura 11: Variación de la intensidad turbulenta longitudinal, medida con el anemómetro de coperolas del mástil a 80 m, a lo largo del día para la dirección de máxima interferencia del aerogenerador A7 comparado con A8. El conjunto de datos se separó entre los meses de invierno (JJA) y verano (DEF).

un nuevo enfoque al estudiar su dependencia con la estabilidad de la CLA. Para los casos de interferencia entre aerogeneradores cercanos, la condición de atmósfera inestable, típica durante el medio día en situaciones con marcado ciclo diurno, inducen una mayor mezcla disipando más rápidamente la estela y atenuando significativamente su impacto en la producción. Particularmente para los casos de medio día en verano, el efecto de estela se reduce casi en su totalidad. Contrariamente, para atmósferas estables, que ocurren generalmente durante la noche, la mezcla de la estela disminuye y permite que se mantenga intensa por mayores distancias, aumentando su impacto. Durante el invierno, la variabilidad en el fenómeno de estela a lo largo del ciclo diurno se ve suavizada.

A pesar de esta dependencia de la estela, y por lo tanto de la eficiencia total del parque, con los regímenes de estabilidad de la CLA, es una práctica normal en la industria analizar la producción de los parques apriori considerando un comportamiento promedio diario y anual 
cercano a la neutralidad. Esta práctica se justifica en el mayor esfuerzo computacional que requiere el uso de herramientas donde se consideren los diferentes regímenes de estabilidad, junto con el mayor conocimiento de las condiciones locales del recurso. En el caso de PER, el estudio de las mediciones en el mástil en la ubicación del parque eólico muestra que en más del $50 \%$ del tiempo la capa de atmósfera cercana a los aerogeneradores es neutral, caracterizada por una turbulencia de origen principalmente mecánico debida a la interacción con la rugosidad del suelo. En este caso, además, la frecuencia con que se observan condiciones estables es ligeramente mayor a la de las inestables, y si bien los efectos de ambas situaciones no se contrarrestan, el resultado final observado se asemeja notablemente al neutral. Este comportamiento podría no generalizarse a otras ubicaciones geográficas, pero parece representar adecuadamente las ubicaciones cercanas al PER en una de las regiones con mejor recurso eólico de la Patagonia Argentina.

Agradecimientos: Los autores desean agradecer a la Agencia Nacional de Promoción de la Investigación, el Desarrollo Tecnológico y la Innovación [PICT2013-1338], al Consejo Nacional de Investigaciones Científicas y Técnicas [PIP 11220120100480CO y becas doctorales GPND y MLM], a la empresa GENNEIA S.A. por facilitar la información del Parque Eólico Rawson y al tiempo de cómputo en el Clúster TUPAC brindado por el CSC-CONICET.

\section{REFERENCIAS}

Bodine, D., Klein, P. M., Arms, S. C., y Shapiro, A. (2009). Variability of surface air temperature over gently sloped terrain. Journal of Applied Meteorology and Climatology, 48(6):1117-1141.

Cañadillas, B., Muñoz-Esparza, D., y Neumann, T. (2011). Fluxes estimation and the derivation of the atmospheric stability at the offshore mast FINO1. En Scientific
Proceedings of the 2011 European Wind Energy Association Offshore Conference and Exhibition, EWEA Offshore'11. EWEA European Wind Energy Association.

Cúneo, L. M., Cerne, S. B., y Llano, M. P. (2018). Descripción preliminar de la velocidad y dirección del viento medio mensual en Trelew. Meteorológica, 44(1):66-80.

Dörenkämper, M., Witha, B., Steinfeld, G., Heinemann, D., y Kühn, M. (2015). The impact of stable atmospheric boundary layers on wind-turbine wakes within offshore wind farms. Journal of Wind Engineering and Industrial Aerodynamics, 144:146-153.

Doubrawa, P., Debnath, M., Moriarty, P. J., Branlard, E., Herges, T., Maniaci, D., y Naughton, B. (2019). Benchmarks for model validation based on lidar wake measurements. En Journal of Physics: Conference Series, volumen 1256, pp 012024. IOP Publishing.

Han, X., Liu, D., Xu, C., y Shen, W. Z. (2018). Atmospheric stability and topography effects on wind turbine performance and wake properties in complex terrain. Renewable energy, 126:640-651.

Mauritsen, T. y Svensson, G. (2007). Observations of stably stratified shear-driven atmospheric turbulence at low and high richardson numbers. Journal of the atmospheric sciences, 64(2):645-655.

Navarro Diaz, G. P., Saulo, A. C., y Otero, A. D. (2019). Wind farm interference and terrain interaction simulation by means of an adaptive actuator disc. Journal of Wind Engineering and Industrial Aerodynamics, 186:58-67.

Newman, J. y Klein, P. (2014). The impacts of atmospheric stability on the accuracy of wind speed extrapolation methods. Resources, 3(1):81-105.

Schepers, J., Obdam, T., y Prospathopoulos, J. (2012). Analysis of wake measurements from the ecn wind turbine test site wieringermeer, ewtw. Wind Energy, 15(4):575-591.

Sedefian, L. y Bennett, E. (1980). A comparison of turbulence classification 
schemes. Atmospheric Environment (1967), 14(7):741-750.

Seguro, J. y Lambert, T. (2000). Modern estimation of the parameters of the weibull wind speed distribution for wind energy analysis. Journal of wind engineering and industrial aerodynamics, 85(1):75-84.

Stull, R. B. (2000). Meteorology for scientists and engineers. Brooks/Cole.

Waimann, C. (2016). Desarrollo de un sistema de pronóstico estocástico-dinámico de producción de energía eólica basado en el modelo WRF/CIMA. Tesis doctoral, Facultad de Ciencias Exactas y Naturales, Universidad de Buenos Aires.

Wharton, S. y Lundquist, J. K. (2012). Assessing atmospheric stability and its impacts on rotor-disk wind characteristics at an onshore wind farm. Wind Energy, 15(4):525-546. 